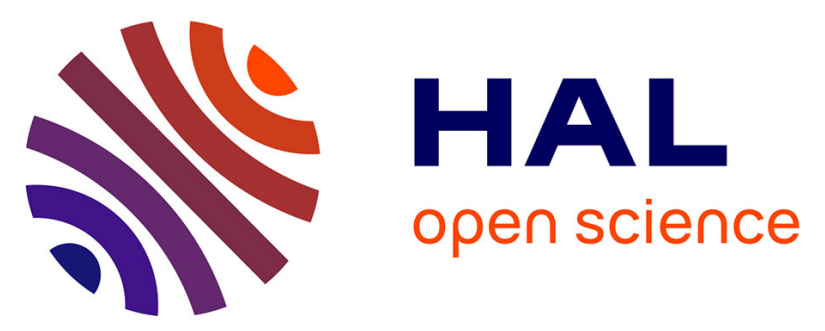

\title{
Screening diversity and distribution of Copia retrotransposons reveals a specific amplification of BARE1 elements in genomes of the polyploid Hordeum murinum complex
}

Malika Ourari, Olivier Coriton, Guillaume Martin, Virginie Huteau, Jean

Keller, Malika-Lily Ainouche, Rachid Amirouche, Abdelkader Ainouche

\section{To cite this version:}

Malika Ourari, Olivier Coriton, Guillaume Martin, Virginie Huteau, Jean Keller, et al.. Screening diversity and distribution of Copia retrotransposons reveals a specific amplification of BARE1 elements in genomes of the polyploid Hordeum murinum complex. Genetica, 2020, 148 (2), pp.109-123. 10.1007/s10709-020-00094-3 . hal-02634590

HAL Id: hal-02634590

https://hal-univ-rennes1.archives-ouvertes.fr/hal-02634590

Submitted on 22 Jun 2020

HAL is a multi-disciplinary open access archive for the deposit and dissemination of scientific research documents, whether they are published or not. The documents may come from teaching and research institutions in France or abroad, or from public or private research centers.
L'archive ouverte pluridisciplinaire HAL, est destinée au dépôt et à la diffusion de documents scientifiques de niveau recherche, publiés ou non, émanant des établissements d'enseignement et de recherche français ou étrangers, des laboratoires publics ou privés. 


\author{
Author Accepted Manuscript \\ "The final publication is available at link.springer.com" \\ Genetica (2020) 148:109-123 \\ https://doi.org/10.1007/s10709-020-00094-3 \\ Published online: 24 April 2020
}

\title{
Screening diversity and distribution of Copia retrotransposons reveals a specific amplification of BARE1 elements in genomes of the polyploid Hordeum murinum complex
}

\author{
Malika Ourari ${ }^{1}$, Olivier Coriton ${ }^{2}$, Guillaume Martin ${ }^{3,7}$, Virginie Huteau ${ }^{2}$, Jean Keller ${ }^{4}$, Malika- \\ Lily Ainouche ${ }^{5}$, Rachid Amirouche ${ }^{6 *}$, Abdelkader Ainouche ${ }^{5}$ \\ ${ }^{1}$ Laboratory of Ecology and Environment, Department of Environment Biological Sciences, Faculty of Nature and Life \\ Sciences, Université de Bejaia, Targa Ouzemmour, 06000 Bejaia, Algeria. \\ ${ }^{2}$ Institut National de Recherche en Agriculture, Alimentation et Environnement, UMR1349 INRAE-AgroCampus Ouest- \\ Université de Rennes 1, Bât 301, INRA Centre de Bretagne-Normandie, BP 35327, 35653 Le Rheu Cedex, France. \\ ${ }^{3}$ CIRAD, UMR AGAP, F-34398 Montpellier, France. \\ ${ }^{4}$ LRSV, Université de Toulouse, CNRS, UPS, 24 chemin de Borde Rouge, Auzeville, 31320 Auzeville-Tolosane, France. \\ ${ }^{5}$ Université de Rennes 1, UMR-CNRS 6553, EcoBio, Campus Scientifique de Beaulieu, Bât. 14A, 35042 Rennes Cedex, \\ France. \\ $6^{6 *}$ University of Sciences and Technology Houari Boumediene, Faculty of Biological Sciences, Lab. LBPO, USTHB, BP 32 \\ El-Alia, Bab-Ezzouar, 16111, Algiers, Algeria \\ ${ }^{7}$ AGAP, Univ Montpellier, CIRAD, INRA, Montpellier SupAgro, Montpellier, France.
}

\section{Corresponding author}

Rachid Amirouche : ramirouche@usthb.dz

\begin{abstract}
We explored diversity, distribution and evolutionary dynamics of Ty1-Copia retrotransposons in the genomes of the Hordeum murinum polyploid complex and related taxa. Phylogenetic and fluorescent in situ hybridization (FISH) analyses of reverse transcriptase sequences identified four Copia families in these genomes: the predominant BARE1 (including three groups or subfamilies, $A, B$ and $C$ ), and the less represented RIRE1, IKYA and TAR-1. Within the BARE1 family, BARE1-A elements and a subgroup of BARE1-B elements (named $B 1$ ) have proliferated in the allopolyploid members of the $H$. murinum complex (H. murinum and $H$. leporinum), and in their extant diploid progenitor, subsp. glaucum. Moreover, we found a specific amplification of BARE1-B elements within each Hordeum species surveyed. The low occurrence of RIRE1, IKYA and TAR-1 elements in the allopolyploid cytotypes suggests that they are either weakly represented or highly degenerated in their diploid progenitors. The results demonstrate that BARE1-A and BARE1-B1 Copia elements are particularly well represented in the genomes of the $H$. murinum complex and constitute its genomic hallmark. No $B A R E 1-A$ and $-B 1$ homologs were detected in the reference barley genome. The similar distribution of RT-Copia probes across chromosomes of diploid, tetraploid and hexaploid taxa of the murinum complex shows no evidence of proliferation following polyploidization.
\end{abstract}

Keywords Hordeum, transposable elements, phylogeny, in situ hybridization, polyploidy 


\section{Introduction}

Transposable elements represent an important and dynamic component of plant genomes (Kumar and Bennetzen 1999; Bennetzen et al. 2005). The most important group of transposable elements (TE) is composed of Long Terminal Repeat (LTR) retrotransposons, which includes two super families named Ty1-Copia and Ty3-Gypsy. These LTR retrotransposons are able to copy themselves through a replicative transposition process (via a RNA intermediate) and then are able to move to new chromosomal locations in the genome (Kumar and Bennetzen 1999). This mode of replication gives retrotransposons the potential to become extremely abundant in plant genomes over time, which increases the size of the host genome. In vascular plants, they can constitute more than half of the repetitive DNA and are generally widely distributed and dispersed in the genomes (Schnable et al. 2009). They are dynamic genome components with the ability to integrate new copies and facilitate intra-chromosomal recombination, and thus can cause large chromosomal rearrangements (Belyayev et al. 2010). Their insertion in close proximity to or into genes may greatly affect gene expression and function via gene disruption and/or TE-derived regulatory sequences capture (Chénais et al. 2012; and references therein). Hence, retrotransposons are an important source of genetic and phenotypic diversity in plants (Kumar and Bennetzen 1999; Mansour 2007; Lisch 2013). Transposition may be activated by various kinds of stress, including genomic shocks (e.g., hybridization, allopolyploidization) and or environmental stimuli (e.g., biotic and abiotic stress) (Granbastien 2004; Kalendar et al. 2000; Liu and Wendel 2003; Parisod et al. 2010). However, this process of transposition is controlled by different and complementary molecular and cellular mechanisms, such as: DNA methylation; miRNA and siRNA; sequence recombination and deletion. This leads to the repression of the TE proliferation and more or less rapidly leads to DNA loss and genome downsizing (Bennetzen et al. 2005; Ma et al. 2004; Ma and Bennetzen 2006; Hawkins et al. 2006, 2009; Yaakov and Kashkush 2011; Lisch and Bennetzen 2011; Chénais et al. 2012).

As a result of these various processes acting repeatedly on TEs dynamics (amplification/repression/recombination/removal) during evolution of species, plant genomes may contain various kinds of TE sequences. Therefore, TEs are represented by full length or truncated elements, likely resulting from very recent amplification, and/or by more or less degenerated but still identifiable TE fragments of various lengths, representing the witnesses of earlier amplifications that occurred in the last 5-10 million years (Devos et al. 2002; Hawkins et al. 2009; Estep et al. 2013). Otherwise, it is generally difficult even impossible to recognize highly degenerated fragments resulting from much older amplification events.

Additionally, hybridization and polyploidy have considerably complexified the impact of these processes over the evolutionary time scales (Ozkan et al. 2001; Parisod et al. 2009). Exploring the diversity and distribution of TE sequences in polyploid and their putative diploid progenitors may provide interesting insights into their evolutionary dynamics in polyploid complexes.

Hordeum L. (Poaceae) is notorious for its large genome and its high TE content. The cultivated barley, $H$. vulgare (diploid with a haploid genome size of $\mathrm{C}=5.1 \mathrm{~Gb}$ ) genome was found containing approximately $84 \%$ of repetitive DNA (Mayer et al. 2012), mainly composed of long terminal repeat (LTR) retrotransposons $(\sim 76 \%)$ belonging to the two LTR-superfamilies, Ty3-Gypsy and Ty1-Copia elements (Suoniemi et al. 1996; Vicient et al. 1999; Mayer et al. 2012). Among the latter, the BARE1 Copia family, which was the first LTR retrotransposon described in barley (Manninen and Schulman 1993 ), is represented by around 80,000 to 120,000 copies, accounting for $12 \%$ of the genome (Wicker et al. 2008, 2009). Examination of BARE1 copy number, conservation and activity in various Hordeum species demonstrated their overall importance to genome evolution in the genus (Vicient et al. 1999). Other known transposable elements and novel repetitive sequences were detected in barley using high throughput sequencing data (Wicker et al. 2009). Polyploidy occurred independently several times in this genus, which offers opportunities to investigate the evolution of TEs throughout closely related members in polyploid groups. In this respect, the H. murinum L. polyploid complex, native from the Mediterranean region, appeared as an interesting group to explore (Vicient et al. 1999; Ourari et al. 2011). 
The Hordeum murinum complex is a monophyletic group composed of one diploid subspecies (subsp. glaucum, $2 n=2 x=14$ ), two tetraploid subspecies (subsp. leporinum, $2 n=4 x=28$; subsp. murinum, $2 n=4 x=28$ ) and one hexaploid subspecies (subsp. leporinum, $2 n=6 x=42$ ) (Love and Love 1948; Covas 1949). Previous studies suggested an allotetraploid origin for subsp. leporinum and subsp. murinum involving the diploid subsp. glaucum (referred to as genome A) as one of their progenitors (the other being unknown or extinct, referred to as genome B). The subspecies leporinum (6x) would have an allohexaploid origin involving genomes A, B and another unidentified diploid parent (referred to as genome C) (Ourari et al. 2011; Brassac and Blattner 2015). The 2C genome size in the "murinum complex" ranged from $\sim 8 \mathrm{pg}$ in the extant diploid progenitor (H. murinum subsp. glaucum) to $17-20 \mathrm{pg}$ in the tetraploid subspecies (leporinum and murinum), and reaches 26 to $30 \mathrm{pg}$ in the hexaploid subsp. leporinum, according to Jakob and Blattner (2010) and Ourari et al. (2011).

In this study, we focus our investigations on the pattern of diversity and distribution of the Copialike LTR-retrotransposons in the H. murinum complex, in order to shed light on the origin of the most remarkable elements detected in the tetra- and hexaploid genomes. Special attention is directed to evaluating the BARE1 family, the best known and most abundant transposable elements in Hordeum (Suoniemi et al. 1996; Vicient et al. 1999; Wicker et al. 2009), which are currently classified within the large Angela group, one of the major evolutionary lineages of Copia elements in angiosperms (Wicker and Keller 2007; Neumann et al. 2019). For this purpose, we used both phylogenetic analyses and genomic fluorescent in situ hybridization (FISH) based on Copia reverse transcriptase conserved domains.

\section{Materials and Methods}

\section{Plant material and DNA extraction}

Two samples of the diploid subsp. glaucum originating from Algeria (M4) and Turkey (gT, USDA collection, plant ID: 402) were analysed. Tetraploid samples of subsp. leporinum and subsp. murinum were collected in Algeria (Tikjda) and France (Brittany), respectively. The hexaploid cytotype analysed was collected in Afghanistan (USDA collection, plant ID: 12910). Representatives of related diploid Hordeum species, Hordeum marinum, $H$. spontaneum and $H$. bulbosum, were sampled in Algeria (Guelt Stel, Sétif and Adekar, respectively).

Genomic DNA was extracted from $100 \mathrm{mg}$ fresh young leaves following the CTAB method adapted from Doyle and Doyle (1987).

\section{Amplification, cloning and sequencing of reverse transcriptase fragments}

Reverse transcriptase ( $R T$ ) sequences of Ty1-Copia elements were amplified using degenerate primers defined by Hirochika and Hirochika (1993) in rice:

TylH1 [5'-CA(A/G)ATGGA(C/T)GT(ACG/T)AA(A/G)AC-3'] and

Ty 1 H 2 [5'- CAT(A/G)TC(A/G)TC(ACG/T)AC(A/G)TA-3'].

PCR conditions were as follows: $3 \mathrm{~min}\left(94^{\circ} \mathrm{C}\right),\left[50 \mathrm{sec}\left(94^{\circ} \mathrm{C}\right), 50 \mathrm{sec}\left(39^{\circ} \mathrm{C}\right), 1 \mathrm{~min}\left(72^{\circ} \mathrm{C}\right)\right] \times 35,7$ $\min \left(72^{\circ} \mathrm{C}\right)$. The PCR products were checked on agarose gel electrophoresis and were purified with a Macherey-Nagel Nucleospin kit. The purified PCR products from all the diploid and polyploid samples were cloned using pGEM-T vectors and JM109 bacterial strains of Escherichia coli from Promega, according to the manufacturer's procedures. Sequencing was performed by Eurofins MWG company (Germany) using T7 primer. Only clones recognisable as RT-Copia sequences by comparison to reference databases (TREPbase, http://wheat.pw.usda.gov/ITMI/Repeats/; and Repbase, Jurka et al. 2005) having a size larger than $230 \mathrm{bp}$ were retained for subsequent analyses; clones of lower size represent more degenerated fragments that are unusable in phylogenetic analyses. Sequence data have been deposited in GenBank (http://www.ncbi.nlm.nih.gov/) under accession numbers JN135335-JN135475.

In order to evaluate and identify the diversity of Copia elements in the murinum complex, two $R T$ data matrices were constructed. One including $141 R T$ clones, generated from the murinum complex 
samples and related taxa (H. marinum, $H$. bulbosum, and $H$. spontaneum) and the other containing all the above $R T$ clones aligned with annotated $R T$ sequences downloaded from reference databases (TREP and Repbase). DNA sequences alignment was performed using the MUSCLE program (Edgar 2004). Pairwise sequence distances (with gaped sites ignored) were estimated using uncorrected "p" with MEGA-X (Kumar et al. 2018). The two matrices were subjected to Maximum Likelihood phylogenetic analyses. The best-fitted model of sequence evolution for each sequence dataset was determined using JModeltest 62 and Maximum likelihood analyses were then performed with 1000 replicates of bootstrap (Felsenstein 1985) using MEGA X. Each group (or clade) of $R T$-domain sequences was named based on its closest phylogenetic relationship to the $R T$-references, following the Copia annotation used for barley and Triticeae (Suoniemi et al. 1996; Vicient et al. 1999; Wicker and Keller 2007; Wicker et al. 2009). Moreover, in order to supplement this annotation in the light of the wider and unified classification system of plant LTR-retrotransposons recently published, all $R T$ sequences were also examined against a reference database of transposable element protein domains (REXdb Viridiplantae 3.0; Neumann et al. 2019), using the BLASTx+ v2.9.0 (Camacho et al. 2009) with default parameter and setting the maximum of hits per query to 10 .

\section{Retrieving the RTs of BARE1 and RIRE elements in the barley genome}

Additionally, we take advantage of the recent release of the barley genome (Hordeum vulgare genome 462R1 v1; Mascher et al. 2017; Beier et al. 2017), available in the Phytozome database (https://phytozome.jgi.doe.gov/pz/portal.html\#), to verify whether some remarkable $R T$ groups observed in our phylogenetic analyses have been specifically amplified in the $H$. murinum complex (see Results). Thus, all BARE1 ( $A$ and $B 1$ ) and RIRE RT sequences generated in our study were blasted against the barley genome of reference, using the BLASTn+ v2.6.3 algorithm. About 125,000 sequences were identified through a BLAST analysis and were clustered with CD-HIT-EST v4.6.8 (Li and Godzik 2006; Fu et al. 2012) and the following parameters: $90 \%$ of identity and a word size of 5. For each of the six obtained cluster, only 19,967 sequences having a minimum length of $270 \mathrm{bp}$ that are aligned with our $R T$ sequences have been retained. Finally, few representatives of these clusters of reference were combined in a same dataset with our BARE1 ( $A$ and B1) and RIRE RT sequences for phylogenetic analysis. Sequences were aligned using MAFFT as implemented in Geneious v6.1.8 (Katoh et al. 2002; Kearse et al. 2012) and then subjected to Maximum Likelihood analysis using IQTREE v1.5 (Nguyen et al. 2015). The best-fitted evolution model was determined with ModelFinder (Kalyaanamoorthy et al. 2017). Phylogenetic analysis was performed with 10,000 SH-alrt and Ultrafast bootstraps replicates (Guindon et al. 2010; Hoang et al. 2017).

\section{Fluorescent in situ hybridization}

Probes derived from RT Copia sequences amplified by degenerate primers (Hirochika and Hirochika 1993) from the diploid subspecies glaucum (A genome) is used in fluorescent in situ hybridization technique (FISH) in order to investigate their chromosomal distribution in the different murinum complex cytotypes.

Paralleling the $R T$-FISH experiments, we have also carried out FISH on the same samples with 45S rDNA probe (pTa 71) to serve as control (Ourari et al. 2011). This sequence is a cloned 9-kb EcoRI fragment of a rDNA repeat unit (18S-5.8S-26S genes and spacers) isolated from Triticum aestivum (Gerlach and Bedbrook 1979). The probe was labeled by random priming with Alexa 594 Kit (Invitrogen Life Technology).

In situ hybridization was performed on mitotic chromosomes from roots of seedlings $(\sim 1 \mathrm{~cm})$ obtained from the diploid and polyploid samples analysed in the $H$. murinum complex. The root tips were treated in $\alpha$-bromonaphtalene solution at $4^{\circ} \mathrm{C}$ for $24 \mathrm{~h}$ and fixed with $3 / 1(\mathrm{v} / \mathrm{v})$ ethanol/aceticacid. Root tips were stained in 2\% aceto-carmine solution and squashed in a drop of $45 \%$ acetic acid. Labelled $R T$ sequences were obtained from targeted genomes by PCR amplification with the PCR DIG Probe Synthesis Kit, according to manufacturer's instructions (Roche, Basel, Switzerland). A hybridization mixture was prepared with $25 \mu 1$ of $100 \%$ formamide, $10 \mu 1$ of $50 \%$ dextran sulfate, $5 \mu 1$ of $20 \times \mathrm{SSC}, 0.6 \mu \mathrm{l}$ of SDS $(0.2 \mathrm{~g} / \mathrm{ml}), 10 \mu \mathrm{g}$ of denatured salmon sperm DNA $(100 \mathrm{mg} / \mathrm{ml})$, 
approximately $100 \mathrm{ng}$ of labelled $R T$ sequences, $5 \mu \mathrm{l}$ of labelled $45 \mathrm{~S}$ rDNA (from Alexa ${ }^{594}$, Kit Invitrogen Life Technology), and ultrapure water for a final volume of $50 \mu 1$. Before hybridization, slides were washed in $2 \times \mathrm{SSC}$ and incubated in RNase at $37^{\circ} \mathrm{C}$ for $1 \mathrm{~h}$. Slides were then rinsed out twice for $3 \mathrm{~min}$ in $2 \times \mathrm{SSC}$ at $42^{\circ} \mathrm{C}$, treated with $100 \mathrm{ml}$ of pepsine at $100 \mu \mathrm{g} / \mathrm{ml} \mathrm{during} 10 \mathrm{~min}$ at $37^{\circ} \mathrm{C}$, rinsed out for $3 \mathrm{~min}$ in $2 \times \mathrm{SSC}$ at $42^{\circ} \mathrm{C}$ (step repeated twice), and plunged in a solution of paraformaldhyde $(0.05 \mathrm{~g} / \mathrm{ml})$ and $\mathrm{NaOH}(0.01 \mathrm{M})$ during $10 \mathrm{~min}$. Slides were rinsed out twice for 3 min in $2 \times \mathrm{SSC}$ at $42^{\circ} \mathrm{C}$, plunged $10 \mathrm{~min}$ in $50 \%$ formamide and dehydrated in a graded ethanol series $(70 \%, 90 \%, 100 \%)$ for $3 \mathrm{~min}$ each in ice-cold. Slides were air-dried at room temperature during 45 min. After a pre-denaturation step $\left(6 \mathrm{~min}\right.$ at $\left.92^{\circ} \mathrm{C}\right), 50 \mu 1$ of the hybridization mixture was applied to the slides. Chromosome preparations and pre-denatured probes were denatured at $85^{\circ} \mathrm{C}$ for $10 \mathrm{~min}$ and allowed to hybridize overnight at $37^{\circ} \mathrm{C}$ in a moist chamber. Slides were treated with $200 \mathrm{ml}$ of $5 \%$ Bovine Serum Albumine in $4 \times$ SSC Tween 20 and incubated for $5 \mathrm{~min}$ at room temperature. To detect the hybridization signal, $50 \mu \mathrm{l}$ of a mixture containing $7 \mu 1$ of Anti-Dig-FITC at $200 \mu \mathrm{g} / \mathrm{ml}$ (Roche) and $63 \mu \mathrm{l}$ of $5 \% \mathrm{BSA}$ were added to the slides. After $1 \mathrm{~h}$ at $37^{\circ} \mathrm{C}$, the slides were washed 3 times in 4 $\times$ SSC for 5 min each at $37^{\circ} \mathrm{C}$. Numerous cells were examined for each hybridization and two independent hybridizations were made for each taxon.

The chromosomes were mounted and counterstained in Vectashield (Vector Laboratories) containing $2.5 \mu \mathrm{g} / \mathrm{mL}$ 4', 6-diamidino-2-phenylindole (DAPI). Fluorescence images were captured using a CoolSnap HQ camera (Photometrics, Tucson, Arizona) on an Axioplan 2 microscope (Zeiss, Oberkochen, Germany) and analyzed using MetaVueTM (Universal Imaging Corporation, Downington, PA).

\section{Results}

\section{RT-Copia diversity in the H. murinum complex}

A total of $141 R T$ sequences of Ty1-Copia elements were obtained from all the taxa and were aligned in a data matrix. Ninety-two clones were generated from the H. murinum complex, namely 27 clones from subsp. glaucum, 23 from the tetraploid subsp. leporinum, 14 from subsp. murinum and 28 from the hexaploid subsp. leporinum. Moreover, 49 clones were generated to represent Copia elements of the closest relatives to the $H$. murinum complex, 26 from $H$. marinum, 11 from $H$. spontaneum and 12 from $H$. bulbosum. The length of this aligned matrix was $297 \mathrm{bp}$. Individual RT Copia fragments ranged from 232 to $279 \mathrm{bp}$ in size. Uncorrected pairwise divergence (p) among the analyzed sequences varied from 0.0 to 0.466 .

As our study is only focused on closely related taxa with highly similar elements, and to allow easier comparison with previously described Copia families, the annotation of the $R T$-domain sequences was essentially based on the classification used for barley and Triticeae (Suoniemi et al. 1996; Vicient et al. 1999; Wicker and Keller 2007; Wicker et al. 2009). A preliminary phylogenetic analysis was performed on the aligned data matrix which included the $141 R T$ sequences generated from this study and annotated $R T$ sequences representing 32 Copia families and subfamilies obtained from TREP and Repbase, in order to identify the Copia diversity in the 'murinum' complex. A simplified ML tree (using the T92+G model; Tamura 1992) is presented in Fig. 1, which includes only one $R T$ reference to represent each of the Copia families and subfamilies detected in our sampling. All cloned sequences fell within four main clusters for which were detected homologs corresponding to the following Copia families: BARE1 (including its close relatives Angela and WIS elements; not shown here), RIRE1, IKYA (incl. its close relative IDA elements; not shown here) and TAR-1 (incl. its close relative SASANDRA elements; not shown here).

Most of the sequences obtained from the murinum complex (78 clones; $84.8 \%$ ) clearly belong to $B A R E 1$, the predominant family described in barley, which is also present in wheat under WIS and Angela names (Wicker and Keller 2007; Wicker et al. 2009). Pairwise divergence (p) among the sequences in the BARE1 cluster varied from 0.0 to 0.305 . Within this family, the 'murinum' sequences were distributed in three main groups or subfamilies $(A, B, C)$ (Fig. 2). BARE1-A, $B$ and $C$ groups are identified by RLC_BARE1_A_consensus1, RLC_BARE1_B_consensus1 and RLC_BARE1_C_consensus1 obtained from reference databases TREP and Repbase, respectively. 
Five $R T$ clones from $H$. murinum subsp. murinum $(4 x)$ and seven from $H$. murinum subsp. leporinum (6x) showed a high homology with RIRE1 elements, a family initially identified in Oryza australiensis (Noma et al. 1997). A single clone from the hexaploid subspecies leporinum is related to family TAR-1 (identified in Triticum monoccocum and homolog to SASANDRA elements from Triticum aestivum), and a single one from the tetraploid subspecies leporinum is related to IKYA (identified in $H$. vulgare) which is homolog to $I D A$ elements from Triticum turgidum. No sequence analyzed in our samples has been related to other much less abundant Copia families (such as MAXIMUS, INGA or CLAUDIA) reported in barley (Wicker et al. 2009).

Therefore, four Copia families have been identified in genomes of the $H$. murinum complex and closely related taxa, BARE1 (including three groups, provisionally treated here as subfamilies $A, B$ and C), RIRE1, IKYA and TAR-1, which are indicated in Fig. 1 and reported in Table 1 (with reference to their homolog families in Triticeae). While adopting this specific classification throughout this study, comparison of our data against the recently published REXdb database, allowed to best situate these families among the main evolutionary lineages of Copia elements circumscribed over a wide range of taxa in the unified classification system of plant LTR-retrotransposons (Neumann et al. 2019). Accordingly, BARE1 and RIRE1 elements (and their homologs WIS and Angela) are supported as members of a same major lineage called "Angela", IKYA (incl. its homolog IDA) is related to the "Ikeros" lineage, whereas TAR-1 is assigned to a lineage with the same name "TAR" (Table 1; Fig. 1).

A second ML analysis (using the T92+G model; Tamura 1992) was conducted on the data matrix only containing the $R T$ sequences generated in this study for the H. murinum complex and allied diploid taxa (Fig.2). Estimates of mean pairwise sequence divergences (i.e., uncorrected 'p-distance') within and among groups of $R T s$ depicted in the tree are given in Table 2. In the large BARE1 clade (supported with $96 \%$ bootstrap), two divergent sequence sets were amplified from the diploid subspecies glaucum ( $2 x$ ): one belonging to BARE1-A subfamily (with $82 \%$ bootstrap support and a Mean Sequence Divergence MSD $=0.111)$; and the other representing part of BARE1-B subfamily, here after named $B A R E 1-B 1$ (with a low bootstrap of $52 \%$ and MSD $=0.098$ ). The mean divergence between these two $R T$ groups ( $A$ vs. $B 1)$ is $\mathrm{p}=0.205$. In addition, $B A R E 1-B$ contained more or less well supported but distinct clusters of sequences that are species-specific to $H$. spontaneum $(92 \%$ of bootstrap support and MDS $=0.047), H$. bulbosum $(52 \%$ bootstrap; MDS $=0.098$ ), and $H$. marinum $(<50 \%$ bootstrap; MDS $=0.089)$, with evolutionary distances among BARE1-B subgroups ranging from 0.107 (between the closely related H. spontaneum and H. bulbosum) to 0.144 (between the $B 1$ and $H$. bulbosum clusters). These two types of $R T$ sequences $(A, B 1)$ are shared by allopolyploids of the murinum complex. The BARE1-C cluster (94\% bootstrap) includes sequences isolated from the polyploid members of the murinum complex (subsp. murinum $4 x$, subsp. leporinum $4 x$, subsp. leporinum $6 x$ ) and others isolated from the related diploids (marinum, spontaneum, bulbosum). None of the sequences cloned from the diploid subsp. glaucum was detected in this cluster (BARE1-C) in which the mean pairwise sequences distance is $\mathrm{p}=0.122$. $R T$-sequences related to RIRE1-I Copia-type were detected in subspecies murinum (4x) and leporinum (6x). Three RIREI-I sequences were also found in H. marinum. The sister relationships between the RIRE and BARE1 elements (79\%) support their membership as distinct subgroups in the same Angela lineages.

The IKYA group includes one sequence detected in subsp. leporinum $4 x$ and sequences from $H$. marinum, whereas some sequences detected in subsp. leporinum $6 x$ and H. bulbosum are identified as representatives of TAR-1 Copia elements.

Following the screening of the reference barley genome $(H$. vulgare syn. of $H$. spontaneum), we detected six clusters of $R T$ sequences which showed a high sequence identity level with the BAREI ( $A$ and B1) and RIRE RT sequences generated from the H. murinum complex (see methods). A phylogenetic analysis was performed on a dataset including both the later $H$. murinum $R T s$ and representatives of the barley clusters. The results (Fig. 3) show that the overwhelming majority of the barley $R T$ s (representing 19,275 of the 19,967 selected) clearly clustered with the H. spontaneum $/ H$. bulbosum BARE1-B subclade (in green in Fig. 3). Another group of sequences representing a substantial number of barley RTs (611) clustered with their RIRE homologs from $H$. murinum polyploids and $H$. marinum. The representatives of the few remaining barley $R T$ s are related to divers other BARE1-B RTs (from H. marinum, H. spontaneum, H. bulbosum and H. leporinum). Therefore, it can be underlined that none of the barley RTs felt within the BARE1-A subfamily or the BARE1-BI 
cluster (respectively in red and blue in Fig. 3), which then likely represent BARE1 elements that evolved and proliferated specifically in the $H$. murinum complex. Also, it is interesting to notice that they neither felt within the specific H. marinum BARE1-B cluster (in purple in Fig. 3).

\section{Distribution of $R T$ elements on the chromosomes of the $H$. murinum complex}

The Copia element probe derived from the subspecies glaucum $(2 x)$ was hybridized to metaphase chromosomes of the murinum complex subspecies to investigate their distribution in the genomes. The probe was hybridized to chromosomes of subspecies glaucum $(2 x)$ itself (Fig. 4a), subspecies leporinum (4x) (Fig. 4b), subspecies тиrinum (4x) (Fig. 4c) and subspecies leporinum (6x) (Fig. 4d).

In all surveyed taxa, this type of elements gives strong hybridisation signal that was uniformly distributed over all chromosomes, except in the NOR and centromeric regions. Moreover, the 45S labeling control was positive in each case and revealed: four 45S rDNA loci in the diploid subsp. glaucum, six in the two tetraploid taxa subsp. murinum and subsp. leporinum, and twelve loci in the hexaploid subsp. leporinum. No locus number additivity for $45 \mathrm{~S}$ rDNA loci was shown in the tetraploids (Fig. 4e).

For this probe, similar signal intensity was found in both diploid and polyploids, and no remarkable differences of their distribution patterns was revealed among the $2 x$ and $4 x-6 x$ cytotypes nor between the polyploid subgenomes.

\section{Discussion}

A fairly complete and regularly updated database, with more than two hundred TE families, is available for repeated elements in Triticeae (TREP, http://wheat.pw.usda.gov/ITMI/Repeats) (Wicker et al. 2008, 2009). The most studied species in genus Hordeum is H. vulgare (syn. spontaneum; barley) (Vicient et al. 1999; Kalendar et al. 2000; Wicker et al. 2007, 2008), which genome has been recently sequenced and released (Hv 462R1 v.1; Mascher et al. 2017; Beier et al. 2017). Currently, no $R T$-Copia data are available for the murinum complex, which represents a widely distributed group of wild populations of Hordeum native from the Euro-Mediterranean region. This study presents the pattern of Ty1-Copia retrotransposon families detected in genomes of diploid and polyploid members of the H. murinum complex, using an exploratory approach based on PCR-amplified fragments and cloning of their conserved $R T$ domain with universal primers (Hirochika and Hirochika 1993; Matsuoka and Tsunewaki 1996; Alix and Heslop-Harrison 2004), Also samples from their closely related diploid species (H. marinum, H. bulbosum, and H. spontaneum) were surveyed for comparison. Diversity and distribution of the Copia elements detected in these taxa are summarized in Fig. 5 and hereafter discussed. In this figure, the different Copia types are indicated by symbols for each taxon. To give an overview of the underlying evolutionary context of this Copia distribution, the taxa analyzed are placed in their phylogenetic framework, redrawn following information generated by Ourari et al. (2011) and Brassac and Blattner (2015). Also, the ploidy level $(2 x, 4 x$ and $6 x)$ and 2C DNA content (in picograms) are indicated for the H. murinum samples analyzed here, according to Ourari et al. (2011).

\section{Diversity of Copia elements in $\boldsymbol{H}$. murinum}

The use of annotated $R T$ sequences from the reference databases TREP and Repbase allowed us to identify four families belonging to the Copia superfamily of LTR-retrotransposon elements in the murinum complex, according to the specific classification used here for barley and their homologs from Triticeae: BARE1, RIRE1, IKYA, TAR-1 (Table 1; Fig. 5). Comparison to the recent unified classification system of plant LTR-retrotransposons (REdb; Neumann et al. 2019) showed that these families derived from within a few ancient major Copia evolutionary lineages in angiosperms: "Angela" (incl. BARE1, RIRE1), "Ikeros" (incl. IKYA) and "TAR" (incl. TAR-1). While "Angela" appears as the lineage which gave rise to the most abundant Copia family (BARE1) in Hordeum, the other lineages are only poorly represented, which indicates an unequal evolutionary dynamics of the ancestral lineages in these taxa. Also, this was observed in rice (a close relative from Oryzeae) where 
the most abundant Copia elements were generated from "TAR" rather than from "Angela" (Wicker and Keller 2007).

Previous studies clearly showed that a few TE families have reached enormous copy numbers in a way they completely dominate the repetitive genomic compartment, whereas dozens of other described TE families contribute only a few percent to the total genome (e.g., Haas et al. 2009; Wicker et al. 2009). In barley, among the $14 \mathrm{TE}$ families that contributed to $50.31 \%$ of the repeated sequences, the Copia element BARE1 tops the list, contributing more than $12 \%$ to the total genome (Vicient et al. 1999; Kalendar et al. 2000; Wicker et al. 2009). Accordingly, our investigation demonstrated that the overwhelming majority of the $R T$ s detected in the reference barley genome ( $H v 462 \mathrm{R} 1)$ essentially represents $B A R E 1$ elements, in agreement with our exploratory PCR-based results which yielded a majority of BARE1 RTs.

Among the much less important Copia families previously observed in barley (Wicker et al. 2009), only very few elements of IKYA and TAR could be identified based on their Triticum homologs, in spite of the reduced number of clones. The others, such as Maximus, Inga, Claudia were not detected in the samples surveyed here using the degenerate primers. This could be explained by either their real absence in the genomes, their very low copy number, and/or the sequencing depth. Moreover, the presumably universal primers used in this study might be not enough performing to amplify some families, due to specific sequence divergence of their $R T s$ or to their high degeneracy if they are or have been present in the genomes. Further investigation of the repetitive sequences in the H. murinum genomes using even a low-depth highthroughput sequencing (NGS) will provide more information and help addressing these uncertainties and questions (Macas et al. 2007; Wicker et al. 2009; Piednoël et al. 2013; Staton and Burke 2015).

Phylogenetic analysis of the $R T$ sequences generated from this study showed that BARE1 Copia elements diversified into three main phylogenetic groups more or less well supported, provisionally treated here as subfamilies $A, B$, and $C$. Further detailed analyses, including more Copia sequences, and using alignments of separate and concatenated protein domain sequences should be helpful to test and adjust this classification at the subfamily level, if needed. Subfamily ' $A$ ' is particularly well represented in the murinum complex, which suggests that the polyploids ( $4 x$ and $6 x$ ) most likely inherited their BARE1-A elements at least from their extant diploid progenitor, subsp. glaucum (Fig. 5 ). The subfamily ' $B$ ' shows a greater diversity and is amplified in all the taxa analyzed (including the extra-murinum diploids examined here). It is noteworthy that some BARE1-B elements specifically cluster according to their taxonomic origin in three subclades: "murinum complex", "marinum", "bulbosum" and "spontaneum" (Figs. 2 and 5). This result suggests that following their inheritance from a common ancestor, $B A R E 1-B$ elements separately experienced specific waves of amplification after speciation. There is an increasing number of studies illustrating such waves of amplification subsequent to speciation in other taxonomic groups, as for instance in Nicotiana, Gossypium or Orobanchaceae (Grandbastien 2004; Hawkins et al. 2006; Piednoël et al., 2013). Indeed, distribution of transposable element families in their host genomes is not necessarily homogeneous. Some elements are restricted to one or two host species, while others may be distributed in several species and genera of the same plant family; which is obvious from this study and indicates that different evolutionary patterns (i.e., differential lineage-specific amplification of TEs) and mechanisms (accumulation/loss) have shaped the Copia profile during the evolutionary history of each species. Many plant retrotransposons are activated by stress or environmental changes (Granbastien 2004; Parisod et al. 2010). For instance, it has been demonstrated significant BARE1 copy number differences among individuals in a wild population of barley (Hordeum spontaneum), in response to a microgeographical gradient of xericity. High elevation, high exposure to sun-light and dryness were correlated with a highest BARE1 copy number (Kalendar et al. 2000). The environmental history of the host will therefore play a crucial role in the amplification dynamics.

Besides, as these elements are dispersed and inserted in various points throughout the genomes they represent a useful potential source of insertion polymorphism for genetic diversity and phylogenetic estimates (Kalendar et al. 1999, 2011). A recent study using inter-retroelement amplified polymorphism (IRAP) markers in the $H$. murinum complex from Iran revealed patterns of genetic diversity correlated with taxonomic groups, ploidy levels and geographic origin (Sharifi-Rigi et al. 2014). 
The sequences obtained from the glaucum diploid genome were distributed in BARE1-A and BARE1-B1; Figs. 2 and 5) also containing sequences from the tetraploid and hexaploid murinum taxa. Therefore, this study allowed identification of two groups of BAREl elements $(A$ and $B 1)$ that are specific to the $H$. murinum complex. Interestingly, this is supported by our screening of the reference barley genome ( $H v 462 \mathrm{R} 1)$ in which no homologous BARE1-A and -B1 RTs could be detected (Fig. 3). Also, this result lends additional support to subsp. glaucum as one of the parents of the polyploids (Jakob and Blattner 2010; Tanno et al. 2010; Ourari et al. 2011; Cuadrado et al. 2013; Brassac and Blattner 2015). In allopolyploid cotton, most TEs inherited from their parental diploid genomes remain closely related to their respective parental orthologs (Hu et al., 2010). Consequently, the authors were able to determine the parental genome origin of many TE sequences from the allopolyploid genome. The other weakly represented Copia elements only detected in the polyploids of the H. murinum complex via the PCR-based approach (such as for instance, BARE1-C, RIRE1, IKYA and TAR-1) suggest that they may have originated from their unknown (most likely extinct) parent (other than glaucum). However, it cannot be ruled out that these elements might have been present in the glaucum genome before being degenerated and/or eliminated, or yet present at a very low copy number. For example, the PCR-based approach did not reveal traces of RIRE1 RTS in our H. spontaneum (syn. vulgare), whereas our investigations allowed detection of the presence of these weakly represented elements in the reference barley genome, which is likely due to a low depth cloning and sequencing. As mentioned above, generating NGS data on Hordeum complex should allow detecting the potential low copy transposable elements, if they are present. Although BARE1-C, RIRE1, IKYA and TAR-1 elements were found in both $H$. murinum subspecies and other diploid Hordeum species analyzed in this study (H. marinum, H. bulbosum or H. spontaneum), it is unlikely that one of them represents a possible progenitor of the $H$. murinum allopolyploids, according to previous congruent phylogenies based on nuclear genes (see Fig. 5), which provided evidence that these taxa are clearly distinct from the H. murinum complex (Jakob and Blattner 2010; Tanno et al. 2010; Ourari et al. 2011). The latter pattern of relationships found additional support and was more accurately defined, using phylogenomic analysis from second-generation sequencing data (Brassac and Blattner 2015), and molecular cytogenetic evidence (Cuadrado et al. 2013). Besides, the potential activation of TEs in genomes following a genomic stress, such as hybridization, has been demonstrated in different systems, as shown for instance in the Zizania $\times$ Oryza hybrid (Liu and Wendel 2000) and in the cotton and wheat allopolypoids (Kraitshtein et al. 2010). It has been shown that different TE families originating from their parental genomes might be differentially amplified following hybridization/allopolyploidization (Parisod et al. 2010; Kraitshtein et al. 2010). Senerchia et al. (2014) also identified different evolutionary trajectories from TE sequences in wild wheat, as demonstrated by proliferation of Sabine- $R T S$ in particular polyploids, but massive elimination in others.

In this study, no remarkable change in the FISH signal intensity using $R T$ probes (Fig. 4) was detected according to ploidy levels in the murinum complex. Even the FISH method only provides a rough estimate of the Copia abundance and scattering in the genomes, no striking change in Copia element intensity was observed in the murinum polyploids $(4 x, 6 x)$, as compared at least to their extant diploid parental genome (H. murinum subsp. glaucum). This suggests that no significant Copia amplification followed allopolyploidization events in this complex, or alternatively that amplification could have been followed by subsequent mechanisms leading to sequence DNA loss. Interestingly, previous data (indicated in Fig. 5) showed that the tetraploid cytotypes (subsp. murinum and subsp. leporinum), which display a nearly similar genome size, have a larger genome $(+0.75-1 \mathrm{pg}$ of DNA) than the approximate value corresponding to the double of the genome size of their extant diploid progenitor (subsp. glaucum) (Ourari et al. 2011). However, regarding that the genome sizes of the other diploid progenitors of the "murinum" polyploids are unknown, and that only Copia elements (among the other TEs) have been surveyed, no conclusion can be made here on the causes of such eventual genome size increase in the tetraploid cytotypes following allopolyploidization. Further analyses based on NGS data and appropriate bioinfiormatic tools (Novak et al. 2010; Staton and Burke 2015) should allow to get a more complete qualitative and quantitative overview on the role of the different types of repetitive sequences (not only Copia elements) in the eventual genome increase in tetraploids. 
Different situations have been reported regarding the dynamics of TEs in synthetic and natural allopolyploids. For instance, only few changes were observed in experimentally resynthetisized polyploids in Gossypium (Liu et al. 2001), whereas the retroelement content increased three-fold in soybean following polyploidy (Innes et al. 2008). Nevertheless, it has been shown that rearrangements resulting in TE loss might occur early after the allopolyploidization event and might hide the amplification effects (Kraitshtein et al. 2010; Yaakov and Kashkush 2011).

We also observed that the Copia-signal was similarly distributed across all the chromosomes and does not distinguish the three murinum subgenomes, suggesting that the unknown parental subgenomes of the $4 x$ and $6 x$ polyploids already possessed these Copia elements. This also, suggests that these subgenomes are closely related, which agree with previous phylogenetic and cytogenetic evidence (Ourari et al. 2011; Cuadrado et al. 2013; Brassac and Blattner 2015). Cuadrado et al. (2013) were able to go further and to distinguish these subgenomes using appropriate SSR-FISH markers.

\section{Distribution of the Copia elements in the genomes of the $H$. murinum complex}

There is evidence from molecular cytogenetic results that retrotransposons show preferential insertion into some genomic regions. For example, many Ty1-Copia retrotransposons are preferentially found within euchromatic regions. Their insertion in close proximity to or directly into genes greatly affects gene expression and function (Kumar and Bennetzen 1999; Grandbastien et al. 2005; Mansour 2007).

In the H. murinum complex, the diploid RT-Copia probes uniformly label the chromosome arms, except at the sub-telomeric and peri-centromeric regions, where the signal is lacking. This $R T$ distribution appears quite similar to that observed in H. vulgare using Copia / Gypsy annotations and $R T$ domains (Mascher et al. 2017), suggesting few changes in the overall organization of Copia in the genus. This pattern was earlier seen for BARE1 using LTR probes in barley (Suoniemi et al. 1996; Vicient et al. 1999), and is consistent with the evidence generated from whole genome sequencing of barley (Mayer et al. 2012).

Also, this is in accordance with data reported in number of plant species such as rice, maize, wheat, barley and sugarcane, in which the centromeric region rather contain Ty3-Gypsy elements than Copia elements (Hirsch and Jiang 2012). Brandes and co-workers (1997) demonstrated that the Ty1-Copia group is dispersed throughout the euchromatic regions, but absent from regions where specialized tandem repeats are expected to lie, such as centromeres, telomeres, heterochromatin, and the nucleolus organizing region (NOR). Additionally, the retroelements are largely excluded, from centromeric satellite arrays. The data suggest that centromeric satellite arrays are under selection for their function in chromosome movement, much like genic regions (SanMiguel et al. 1996).

In conclusion:

In this study, the diversity of the Copia-like LTR-retrotransposons occurring in the genomes of the H. murinum polyploid complex has been investigated, using a PCR-based approach of their conserved $R T$ domain. In spite of some expected sampling biases and limits of the method (particularly, performance of universal primers, and sequencing depth), the PCR-based exploration provided a first overview on the diversity, distribution and relative importance of Copia elements in the "H. murinum" complex and related taxa.

Therefore, it was shown that Ty1-Copia retroelements are well represented in the genomes of the $H$. murinum L. complex. These elements belong to four Copia families referred to BARE1, RIRE1, IKYA, and TAR-1. In accordance with studies using NGS-based data on barley, BARE1 also appeared as the most important Copia family detected in these genomes. Among the three major subfamilies $(A, B, C)$ found in BARE1, BARE1-B elements showed a great diversity which is represented in all taxa analyzed, including $H$. bulbosum, $H$. spontaneum, $H$. marinum and $H$. murinum. BARE $1-A$ and $B A R E 1-B$ elements appear to have been specifically amplified in the $H$. murinum complex, thus providing a genomic hallmark to this taxonomic group. In contrast, the other BARE1-C, IKYA and $T A R-1$ elements are poorly represented in the murinum polyploid cytotypes and seem absent in the diploid subspecies glaucum. These elements could come from the other two unknown diploid parents of the polyploids or might result from amplification following allopolyploidization events.

In situ hybridization method (FISH) confirmed the abundance and similar distribution of $R T$ elements throughout the diploid and polyploid genomes (excepted in the centromeric and NOR 
regions). No remarkable change in retroelement intensity was detectable in the murinum polyploids ( $4 x$ and $6 x$ ), as compared to their diploid parental genome (H. murinum subsp. glaucum), showing no evidence of Copia amplification following allopolyploidization in the $H$. murinum complex.

Overall, the results are consistent with the main findings observed elsewhere in barley, and provided new information and insights on the Copia elements occurring in this complex. Therefore, this study provides a consistent framework (including questions to be addressed) that paves the way for further deepest NGS-based evaluation of the repetitive content of the $H$ murinum genomes to better understand their evolutionary dynamics.

\section{Acknowledgements}

Both the Algerian and French Ministries of Education and Research and the French-Algerian PHC joint research program are thanked for funding support to our collaborative Project Tassili 08 MDU 724.

\section{Conflict of interest}

The authors declare that they have no conflict of interest.

\section{Data Archiving}

Sequence data have been submitted to GenBank under accession numbers JN135335-JN135475.

\section{References}

Alix K, Heslop-Harrison JS (2004) The diversity of retroelements in diploid and allotetraploid Brassica species. Plant Mol Biol 54(6):895-909. doi:10.1007/s11103-004-0391-Z

Beier S, Himmelbach A, Colmsee C, Zhang X-Q, Barrero RA, Zhang Q et al. (2017) Construction of a map-based reference genome sequence for barley, Hordeum vulgare L. Scientific Data 4(1): 170044-170044. https://doi.org/10.1038/sdata.2017.44

Belyayev A, Kalendar R, Brodsky L, Nevo E, Schulman AH, Raskina O (2010) Transposable elements in a marginal plant population: temporal fluctuations provide new insights into genome evolution of wild diploid wheat. Mobile DNA 1(1): 6. doi:10.1186/1759-8753-1-6

Bennetzen JL, Ma J, Devos KM (2005) Mechanisms of recent genome size variation in flowering plants. Ann Bot 95 (1): 127-132. doi:10.1093/aob/mci008

Brandes A, Heslop-Harrison JS, Kamm A, Doudrick RL, Schmidt T (1997) Comparative analysis of the chromosomal and genomic organization of Ty1-Copia-like retrotransposons in Pteridophytes, Gymnosperms and Angiosperms. Plant Mol Biol 33(1):11-21. doi:10.1023/A:1005797222148

Brassac J, Blattner FR (2015) Species-Level Phylogeny and Polyploid Relationships in Hordeum (Poaceae) Inferred by Next-Generation Sequencing and In Silico Cloning of Multiple Nuclear Loci. Syst Biol 64(5): 792-808. doi:10.1093/sysbio/syv035. PMID:26048340

Camacho C, Coulouris G, Avagyan V, Ma N, Papadopoulos J, Bealer K, Madden TL (2009) BLAST+: architecture and applica-tions. BMC Bioinformatics 10: 421. doi :10.1186/1471-2105$10-421$

Chénais B, Caruso A, Hiard S, Casse N (2012) The impact of transposable elements on eukaryotic genomes: From genome size increase to genetic adaptation to stressful environments. Gene 509(1): 7-15. doi.org/10.1016/j.gene.2012.07.042. PMID: 22921893

Covas G (1949) Taxonomic observations on the North American species of Hordeum. Madrono 10: 121

Cuadrado A, Carmona A, Jouve N (2013) Chromosomal Characterization of the Three Subgenomes in the Polyploids of Hordeum murinum L.: New Insight into the Evolution of This Complex. PloS ONE 8(12). e81385. doi.org/10.1371/journal.pone.0081385

Devos KM, Brown JK, Bennetzen JL (2002) Genome size reduction through illegitimate recombination counteracts genome expansion in Arabidopsis. Genome Res 12(7):1075-1079. doi:10.1101/gr.132102

Doyle JJ, Doyle JL (1987) A rapid DNA isolation procedure for small quantities of fresh leaf tissue. Phytochem Bull 19(1): 11-15 
Edgar RC (2004) MUSCLE: A multiple sequence alignment method with reduced time and space complexity. BMC Bioinformatics 5(1):113. doi:10.1186/1471-2105-5-113

Estep MC, DeBarry JD, Bennetzen JL (2013) The dynamics of LTR retrotransposon accumulation across 25 million years of panicoid grass evolution. Heredity 110(2):194-204. doi:10.1038/hdy.2012.99

Felsenstein J (1985) Confidence limits on phylogenies: an approach using the bootstrap. Evolution 39(4): 783-791. doi:10.2307/2408678

Fu L, Niu B, Zhu Z, Wu S, Li W (2012) CD-HIT: accelerated for clustering the next-generation sequencing data. Bioinformatics 28:3150-3152. Doi:10.1093/bioinformatics/bts565.

Gerlach WL, Bedbrook JR (1979) Cloning and characterization of ribosomal RNA genes from wheat and barley. Nucleic Acids Res 7(7):1869-1885. doi:10.1093/nar/7.7.1869

Grandbastien MA (2004) Stress activation and genomic impact of plant retrotransposons. J Soc Biol 198(4): 425-432. PMID:15969350

Grandbastien MA, Audeon C, Bonnivard E, Casacuberta JM, Chalhoub B, Costa AP et al. (2005) Stress activation and genomic impact of Tnt1 retrotransposons in Solanaceae. Cytogenet Genome Res 110(1-4):229-41. doi:10.1159/000084957

Guindon S, Dufayard JF, Lefort V, Anisimova M, Hordijk W, Gascuel, O (2010) New Algorithms and Methods to Estimate Maximum-Likelihood Phylogenies: Assessing the Performance of PhyML 3.0. Systematic Biology 59(3):307-321. doi:10.1093/sysbio/syq010

Haas BJ, Kamoun S, Zody MC, Jiang RHY, Handsaker RE, Cano LM et al. (2009) Genome sequence and analysis of the Irish potato famine pathogen Phytophthora infestans. Nature 461(7262):3938. doi: 10.1038 /nature 08358

Hawkins JS, Kim H, Nason JD, Wing RA, Wendel JF (2006) Differential lineage-specific amplification of transposable elements is responsible for genome size variation in Gossypium. Genome Res 16(10):1252-1261. doi/10.1101/gr.5282906

Hawkins JS, Proulx SR, Rapp RA, Wendel JF (2009) Rapid DNA loss as a counterbalance to genome expansion through retrotransposon proliferation in plants. Proc Natl Acad Sci USA 106 (42):17811-17816. doi:10.1073/pnas.0904339106

Hirochika H, Hirochika R (1993) Ty1-copia group retrotransposons as ubiquitous components of plant genomes. Jpn J Genet 68(1):35-46. doi:10.1266/ggs.68.35

Hirsch CD, Jiang J (2012) Centromeres: sequences, structure, and biology. In Wendel JF, Greilhuber J, Dolezel J, Leitch IJ et al. (Ed). Plant Genome Diversity, Volume 1: Plant Genomes, Their Residents, and Their Evolutionary Dynamics. Springer-Verlag, Wien, pp 59-70. doi:10.1007/9783-7091-1130-7_4.

Hoang DT, Chernomor O, von Haeseler A, Minh BQ, Le SV (2017) UFBoot2: Improving the ultrafast bootstrap approximation. Mol Biol Evol 35(2):518-522doi:10.1093/molbev/msx281

Hu G, Hawkins JS, Grover CE, Wendel JF (2010) The history and disposition of transposable elements in polyploid Gossypium. Genome 53(8):599-607. doi:10.1139/g10-038

Innes RW, Ameline-Torregrosa C, Ashfield T (2008) Differential accumulation of retroelements and diversification of NB-LRR disease resistance genes in duplicated regions following polyploidy in the ancestor of soybean. Plant Physiol 148(4):1740-1759. doi:10.1104/pp.108.127902

Jakob SS, Blattner FR (2010) Two extinct diploid progenitors were involved in allopolyploid formation in the Hordeum murinum (Poaceae: Triticeae) taxon complex. Mol Phylogenet Evol 55(2):650-659. doi.org/10.1016/j.ympev.2009.10.021

Jurka J, Kapitonov VV, Pavlicek A, Klonowski P, Kohany O, Walichiewicz J et al. (2005) Repbase Update, a database of eukaryotic repetitive elements. Cytogenet Genome Res 110(1-4):462-467. doi:10.1159/000084979

Kalendar R, Flavell AJ, Ellis THN, Sjakste T, Moisy C, Schulman AH (2011) Analysis of plant diversity with retrotransposon-based molecular markers. Heredity 106(4):520-530. doi:10.1038/hdy.2010.93

Kalendar R, Grab T, Regina M, Suoniemi A, Schulman AH (1999) IRAP and REMAP: two new retrotransposon-based DNA fingerprinting techniques. Theor Appl Genet 98(5):704-711. doi: $10.1007 / \mathrm{s} 001220051124$ 
Kalendar R, Tanskanen J, Immonen S, Nevo E, Schulman AH (2000) Genome evolution of wild barley (Hordeum spontaneum) by BARE1 retrotransposon dynamics in response to sharp microclimatic divergence. Proc Natl Acad Sci USA 97(12):6603-6607. doi:0.1073/pnas.110587497

Kalyaanamoorthy S, Minh B, Wong T et al. (2017) ModelFinder: fast model selection for accurate phylogenetic estimates. Nat Methods 14:587-589. https://doi.org/10.1038/nmeth.4285

Katoh K, Misawa K, Kuma K, Miyata T (2002) MAFFT: a novel method for rapid multiple sequence alignment based on fast Fourier transform. Nucleic Acids Res 15-30:3059-66 Nucleic Acids Res. 15;30(14):3059-66

Kearse M, Moir R, Wilson A, Stones-Havas S, Cheung M, Sturrock S et al. (2012) Geneious Basic: an integrated and extendable desktop software platform for the organization and analysis of sequence data. Bioinformatics 15-28(12):1647-9. doi:10.1093/bioinformatics/bts199

Kraitshtein Z, Yaakov B, Khasdan V (2010) The genetic and epigenetic dynamics of a retrotransposon after allopolyploidization of wheat. Genetics 186(3):801-812. doi:10.1534/genetics.110.120790

Kumar A, Bennetzen JL (1999) Plant retrotransposons. Ann Rev Genet 30:479-532. doi:10.1146/annurev.genet.33.1.479

Kumar S, Stecher G, Li M, Knyaz C, Tamura K (2018) MEGA X: Molecular Evolutionary Genetics Analysis across Computing Platforms. Mol Biol Evol 35(6):1547-1549 doi:10.1093/molbev/msy096

Li W, Godzik A (2006) Cd-Hit: a Fast Program for Clustering and Comparing Large Sets of Protein or Nucleotide Sequences. Bioinformatics 22(13):1658-9. DOI:10.1093/bioinformatics/btl158

Lisch D (2013) How important are transposons for plant evolution? Nature Reviews Genetics 14 (1):49-61. doi: $10.1038 / \mathrm{nrg} 3374$

Lisch D, Bennetzen JL (2011) Transposable element origins of epigenetic gene regulation. Curr Opin Plant Biol 14(2):156-161. doi.org/10.1016/j.pbi.2011.01.003

Liu B, Brubaker CL, Mergeai G, Cronn RC, Wendel JF (2001) Polyploid formation in cotton is not accompanied by rapid genomic changes. Genome 44(3):321-330. doi:10.1139/gen-44-3-321

Liu B, Wendel JF (2000) Retroelement activation followed by rapid repression in interspecific hybrid plants. Genome 43(5):874-880. doi :10.1139/g00-058

Liu B, Wendel JF (2003) Epigenetic phenomena and the evolution of plant allopolyploids. Mol Phylogenet Evol 29(3):365-379. doi:10.1016/S1055-7903(03)00213-6

Löve A, Löve D (1948) Chromosome numbers of northern plant species. Dept, of Agr. Report Ser. B, No 3, Iceland.

Ma J, Bennetzen JL (2006) Recombination, rearrangement, reshuffling, and divergence in a centromeric region of rice. Proc Natl Acad Sci USA 103(2):383-8. doi:10.1073/pnas.0509810102

Ma XF, Fang P, Gustafson JP (2004) Polyploidization-induced genome variation in triticale. Genome 47(5):839-848. doi:10.1139/g04-051

Macas J, Neumann P, Navrátilová A (2007) Repetitive DNA in the pea (Pisum sativum L.) genome: comprehensive characterization using 454 sequencing and comparison to soybean and Medicago truncatula. BMC. BMC Genomics 8:427. https://doi.org/10.1186/1471-2164-8-427

Manninen I, Schulman AH (1993) BARE1, a copia-like retroelement in barley (Hordeum vulgare L.). Plant Mol Biol 22(5):829-846. doi:10.1007/BF00027369

Mansour A (2007) Epigenetic activation of Genomic Retrotransposon. J Cell Mol Biol 6(2):99-107

Mascher M, Gundlach H, Himmelbach A, Beier S, Twardziok SO, Wicker T et al (2017) A chromosome conformation capture ordered sequence of the barley genome. Nature 544(427). doi:10.1038/nature22043

Matsuoka Y, Tsunewaki K (1996) Wheat Retrotransposon Families Identified by Reverse Transcriptase Domain Analysis. Mol. Biol. Evol. 13(10):1384-1392

Mayer KF, Waugh R, Brown JW, Schulman A, Langridge P, Platzer M et al. (2012) A physical genetic and functional sequence assembly of the barley genome. Nature 491(7426):711-716. doi:10.1038/nature 11543

Neumann P, Novák P, Hoštáková N, Macas J (2019) Systematic survey of plant LTR retrotransposons elucidates phylogenetic relationships of their polyprotein domains and provides a reference for element classification. Mobile DNA 10:1. https://doi.org/10.1186/s13100-018-0144-1

Lam-Tung Nguyen, Heiko A., Arndt von Haeseler, Bui Quang Author Notes 
Nguyen LT, Schmidt HA, Haeseler A, Minh BQ (2015) IQ-TREE: A fast and effective stochastic algorithm for estimating maximum-likelihood phylogenies. Mol Biol Evol 32(1):268-274. https://doi.org/10.1093/molbev/msu300

Noma K, Nakajima R, Ohtsubo H, Ohtsubo E (1997) RIRE1, a retrotransposon from wild rice Oryza australiensis. Genes Genet Syst 72(3):131-140. doi:10.1266/ggs.72.131

Novak P, Neumann P, Macas J (2010) Graph-based clustering and characterization of repetitive sequences in next-generation sequencing data. BMC Bioinformatics 11: 378

Ourari M, Ainouche A, Coriton O, Huteau V, Brown S, Misset MT et al. (2011) Diversity and evolution of the Hordeum murinum polyploid complex in Algeria. Genome 54(8):639-654. doi:10.1139/g65-040

Ozkan H, Levy AA, Feldman M (2001) Allopolyploidy-induced rapid genome evolution in the wheat (Aegilops-Triticum) group. Plant Cell 13(8):1735-1747. doi:10.1105/TPC.010082

Parisod C, Salmon A, Zerjal T, Tenaillon M, Grandbastien MA, Ainouche M (2009) Rapid structural and epigenetic reorganization near transposable elements in hybrid and allopolyploid genomes in Spartina. New Phytol 184(4):1003-1015. doi:10.1111/j.1469-8137.2009.03029.x

Parisod C, Alix J, Just M, Petit V, Mhiri C, Ainouche M et al. (2010). Impact of transposable elements on the organisation and function of allopolyploid genomes. New Phytol 186(1):37-47. doi:10.1111/j.1469-8137.2009.03096.x

Piednoël M, Carrete-Vega G, Renner SS (2013) Characterization of the LTR retrotransposon repertoire of a plant clade of six diploid and one tetraploid species. The Plant Journal 75: 699-709. doi: $10.1111 /$ tpj. 1223

SanMiguel P, Tikhonov A, Jin YK, Motchoulskaia N, Zakharov D, Melake-Berhan A et al. (1996) Nested retrotransposons in the intergenic regions of the maize genome. Science 274(5288):765768. doi:10.1126/science.274.5288.765

Schnable PS, Ware D, Fulton RS, Stein JC, Wei F, Pasternak S et al. (2009) The B73 maize genome: complexity, diversity, and dynamics. Science 326(5956):1112-1115. doi:10.1126/science.1178534

Senerchia N, Felber F, Parisod C (2014) Contrasting evolutionary trajectories of multiple retrotransposons following independent allopolyploidy in wild wheats. New Phytol 202(3):975985. doi:10.1111/nph.12731

Sharifi-Rigi P, Saeidi H, Rahiminejad MR (2014) Genetic diversity and geographic distribution of variation of Hordeum murinum as revealed by retroelement insertional polymorphisms in Iran. Biologia 69(4):469-477. doi:10.2478/s11756-014-0340-5

Staton SE, Burke JM (2015) Transposome: a toolkit for annotation of transposable element families from unassembled sequence reads Bioinformatics 31:1827-1829. doi: 10.1093/bioinformatics/btv059

Suoniemi A, Anamthawat-Jonsson K, Arna T, Schulman AH (1996) Retrotransposon BARE1 is a major dispersed component of the barley (Hordeum vulgare L.) genome. Plant Mol Biol 30(6):1321-1329. doi:10.1007/BF00019563

Tamura K (1992) Estimation of the number of nucleotide substitutions when there are strong transition-transversion and $\mathrm{G}+\mathrm{C}$ content biases. Mol Biol Evol 9:678-68. DOI: 10.1093/oxfordjournals.molbev.a040752

Tanno K, von Bothmer R, Yamane K, Takeda K, Komatsuda T (2010) Analysis of DNA sequence polymorphism at the cMWG699 locus reveals phylogenetic relationships and allopolyploidy within Hordeum murinum subspecies. Hereditas 147(1):34-42. doi:10.1111/j.16015223.2009.02142.x

Vicient CM, Suoniemi A, Anamthawat-Jónsson K, Tanskanen J, Beharav A, Nevo E et al. (1999) Retrotransposon BARE1 and its role in genome evolution in the genus Hordeum. Plant Cell 11(9):1769-1784. doi:10.1105/tpc.11.9.1769

Wicker T, Keller B (2007) Genome-wide comparative analysis of copia retrotransposon in triticeae, rice, and Arabidopsis reveals conserved ancient evolutionary lineages and distinct dynamics of individual copia families. Genome Res 17(7):1072-1081. doi:10.1101/gr.6214107

Wicker T, Narechania A, Sabot F, Stein J, Giang VTH, Graner A et al. (2008) Low-pass shotgun sequencing of the barley genome facilitates rapid identification of genes, conserved non-coding sequences and novel repeats. BMC Genomics 9: 518. doi:10.1186/1471-2164-9-518 
Wicker T, Sabot F, Hua-Van A, Bennetzen JL, Capy P, Chalhoub B et al. (2007) A unified classification system for eukaryotic transposable elements. Nature Reviews Genetics 8(12):973982. doi: $10.1038 / \mathrm{nrg} 2165$

Wicker T, Taudien S, Houben A, Keller B, Graner A, Platzer M et al. (2009) A whole-genome snapshot of 454 sequences exposes the composition of the barley genome and provides evidence for parallel evolution of genome size in wheat and barley. Plant J 59(5):712-722. doi:10.1111/j.1365313X.2009.03911.X

Yaakov B, Kashkush K (2011) Methylation, Transcription, and Rearrangements of Transposable Elements in Synthetic Allopolyploids. International Journal of Plant Genomics, vol. 2011, Article ID 569826, http://dx.doi.org/10.1155/2011/569826 
Table 1 Annotation of the Copia-like elements detected in the Hordeum murinum L. complex and closely related taxa.

\begin{tabular}{|c|c|c|}
\hline Annotation used in this study ${ }^{a}$ & \multirow[t]{2}{*}{ Homolog families ${ }^{\text {a,b }}$} & \multirow[t]{2}{*}{ REXdb Copia lineage ${ }^{c}$} \\
\hline Family/subfamily & & \\
\hline$B A R E 1-A$ & WIS; Angela & Angela \\
\hline$B A R E 1-B$ & WIS; Angela & Angela \\
\hline$B A R E 1-C$ & WIS; Angela & Angela \\
\hline RIREI & & Angela \\
\hline$I K Y A$ & $I D A$ & Ikeros \\
\hline TAR-1 & SASANDRA & TAR \\
\hline \multicolumn{3}{|c|}{$\begin{array}{l}\text { a Folowing the specific classification for barley and Triticeae (Suoniemi et al. 1996; Vicient et al. 1999; Wicker } \\
\text { et al. 2009) }\end{array}$} \\
\hline \multicolumn{3}{|l|}{${ }^{b}$ From TREP and Repbase } \\
\hline${ }^{c}$ From the unified classification sys & nt LTR-retro & et al. 2019) \\
\hline
\end{tabular}

Table 2 Estimates of average pairwise sequence divergence (p-distance) within (in bold) and between groups of Copia-like elements in the Hordeum murinum L. complex using MEGA X software (Kumar et al. 2018)

\begin{tabular}{|c|c|c|c|c|c|c|c|c|c|}
\hline \multirow{3}{*}{$\begin{array}{l}\text { Groups of Copia } \\
\text { elements }\end{array}$} & \multicolumn{6}{|c|}{$B A R E 1$} & \multirow{3}{*}{ RIRE1 } & \multirow{3}{*}{$I K Y A$} & \multirow{3}{*}{$T A R-1$} \\
\hline & \multirow[b]{2}{*}{$\boldsymbol{A}$} & \multicolumn{4}{|l|}{$B$} & \multirow{2}{*}{$C$} & & & \\
\hline & & $B 1$ & marinum & bulbosum & spontaneum & & & & \\
\hline$B A R E 1-A$ & 0.111 & & & & & & & & \\
\hline$B A R E 1-B 1$ & 0.205 & 0.098 & & & & & & & \\
\hline BARE1-B marinum & 0.196 & 0.121 & 0.089 & & & & & & \\
\hline BARE1-B bulbosum & 0.209 & 0.144 & 0.136 & 0.090 & & & & & \\
\hline BARE1-B spontaneum & 0.201 & 0.126 & 0.133 & 0.107 & 0.047 & & & & \\
\hline$B A R E 1-C$ & 0.231 & 0.167 & 0.171 & 0.171 & 0.151 & 0.122 & & & \\
\hline RIRE1 & 0.358 & 0.311 & 0.326 & 0.292 & 0.288 & 0.296 & 0.098 & & \\
\hline$I K Y A-I D A$ & 0.366 & 0.358 & 0.360 & 0.352 & 0.333 & 0.355 & 0.380 & 0.044 & \\
\hline$T A R-1$ & 0.430 & 0.410 & 0.411 & 0.404 & 0.408 & 0.408 & 0.362 & 0.387 & 0.133 \\
\hline
\end{tabular}




\section{TITLES AND LEGENDS TO FIGURES}

Fig. 1 Maximum likelihood tree (using the T92+G evolutionary model) of 141 reverse transcriptase sequences representing Copia-like retrotransposons cloned in the H. murinum L. complex and its closest diploid relatives, $H$. marinum, $H$. bulbosum and $H$. spontaneum. Bootstrap values higher than $50 \%$ are given for the main nodes. This unrooted tree includes annotated $R T$ sequences from referee databases, TREP and REPbase, which are indicated by arrowed branches. Four families are circumscribed in $\mathrm{t}$ he tree, following the specific Copia classification used for barley and Triticeae.: BARE1, RIRE1-I, IKYA, TAR-1. Also is superimposed in the tree, the lineage assignment of these Copia families according to the recently published unified classification system of plant LTRretrotransposons (Neumann et al. 2019)

Fig. 2 Maximum likelihood tree (using the $\mathrm{T} 92+\mathrm{G}$ evolutionary model) of reverse transcriptase sequences representing Ty1-Copia retrotransposons families identified in the H. murinum complex and their c losely related diploid taxa, $H$. marinum, $H$. spontaneum and $H$. bulbosum. Annotation assignments of the main clades and subclades of interest are indicated in the tree, following the specific Copia classification used for barley and Triticeae.: BARE1 (A, B, C), RIRE1-I, IKYA, TAR-1. Taxonomic origins of the $R T$-clones are given at the right of the tree for each clade. Bootstrap values (as \%) are indicated on the nodes of the main clades

Fig. 3 Maximum likelihood tree generated from the analysis of a dataset including (i) all BARE1 (A and $B 1$ ) and RIRE RT sequences cloned and sequenced in this study from the H. murinum complex, $H$. marinum, $H$. bulbosum and $H$. spontaneum, and (ii) $24 R T$ sequences from the reference barley genome (highlighted in yellow in the tree) representing clusters of retrotransposon which show the highest sequence similarity with the BAREl ( $A$ and $B I$ ) and RIRE RT of the H. murinum complex. Annotation and taxonomic origins of the $R T$-clones are indicated in the tree. None of the barley $R T S$ fell within the BARE1 - $A$ or BARE1-B1 subclades of H. murinum

Fig. 4 FISH karyotype analysis in the H. murinum complex using RT-Copia probe from H. murinum subsp. glaucum (in green) and 45S rDNA (in red). (a): H. murinum subsp. glaucum (2x); (b): H. murinum subsp. leporinum (4x); (c): H. murinum subsp. murinum (4x); (d): H. murinum subsp. leporinum (6x). Chromosomes carrying rDNA are identified in (e). Chromosomes were counterstained with DAPI (blue). Scale bars $=5 \mu \mathrm{m}$

Fig. 5 Distribution of retrotransposon Ty1-Copia subfamilies detected throughout the diploid and polyploid members of the $H$. murinum complex and its close diploid relatives, using amplification with universal primers and cloning of their reverse transcriptase domain. The phylogenetic tree of the Hordeum taxa is redrawn from Ourari et al. (2011) and Brassac and Blattner (2015). 2C DNA content (in picogrammes) is indicated in brackets for the H. murinum samples analyzed here, according to Ourari et al. (2011) 
Fig.1

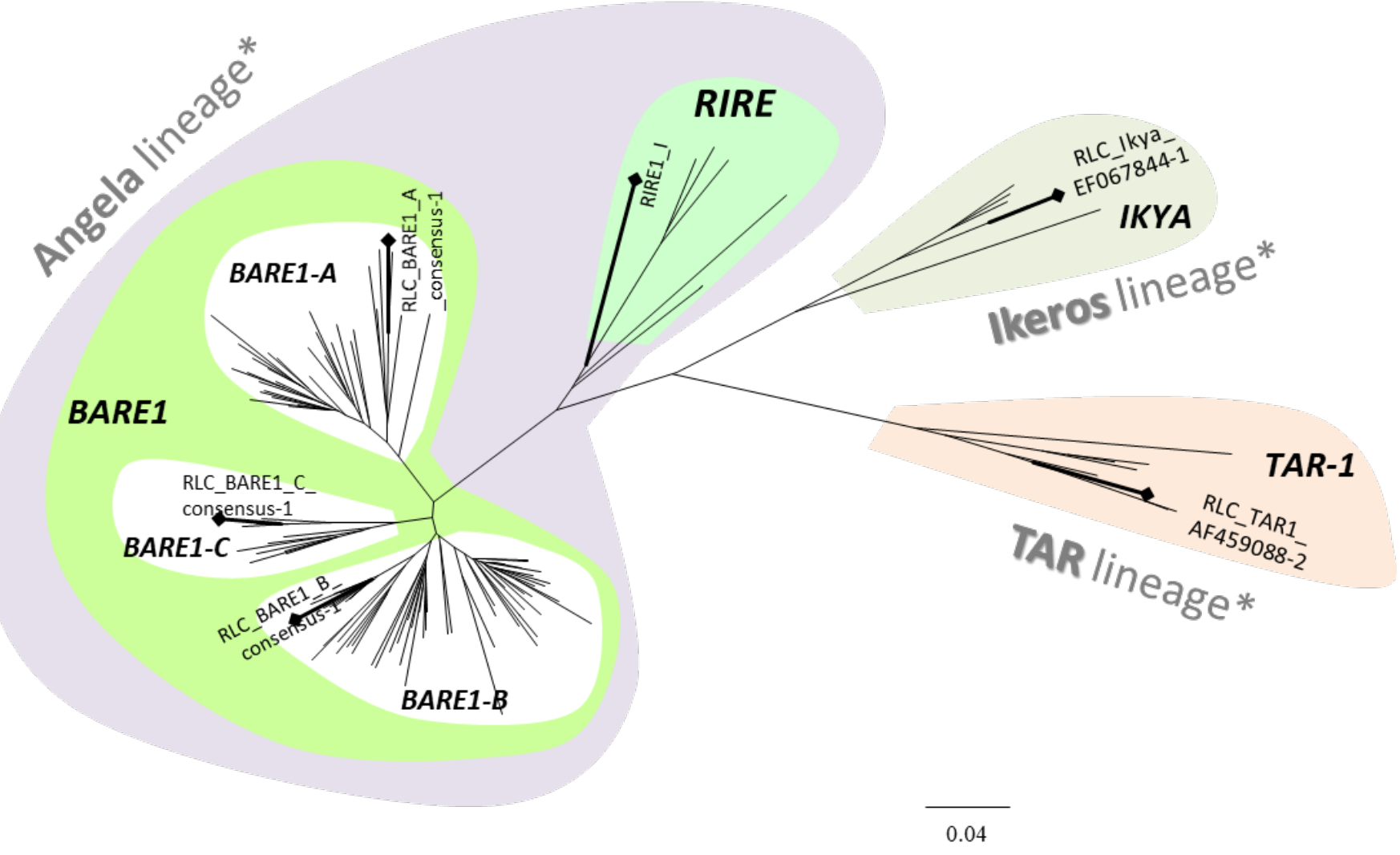


Fig.2

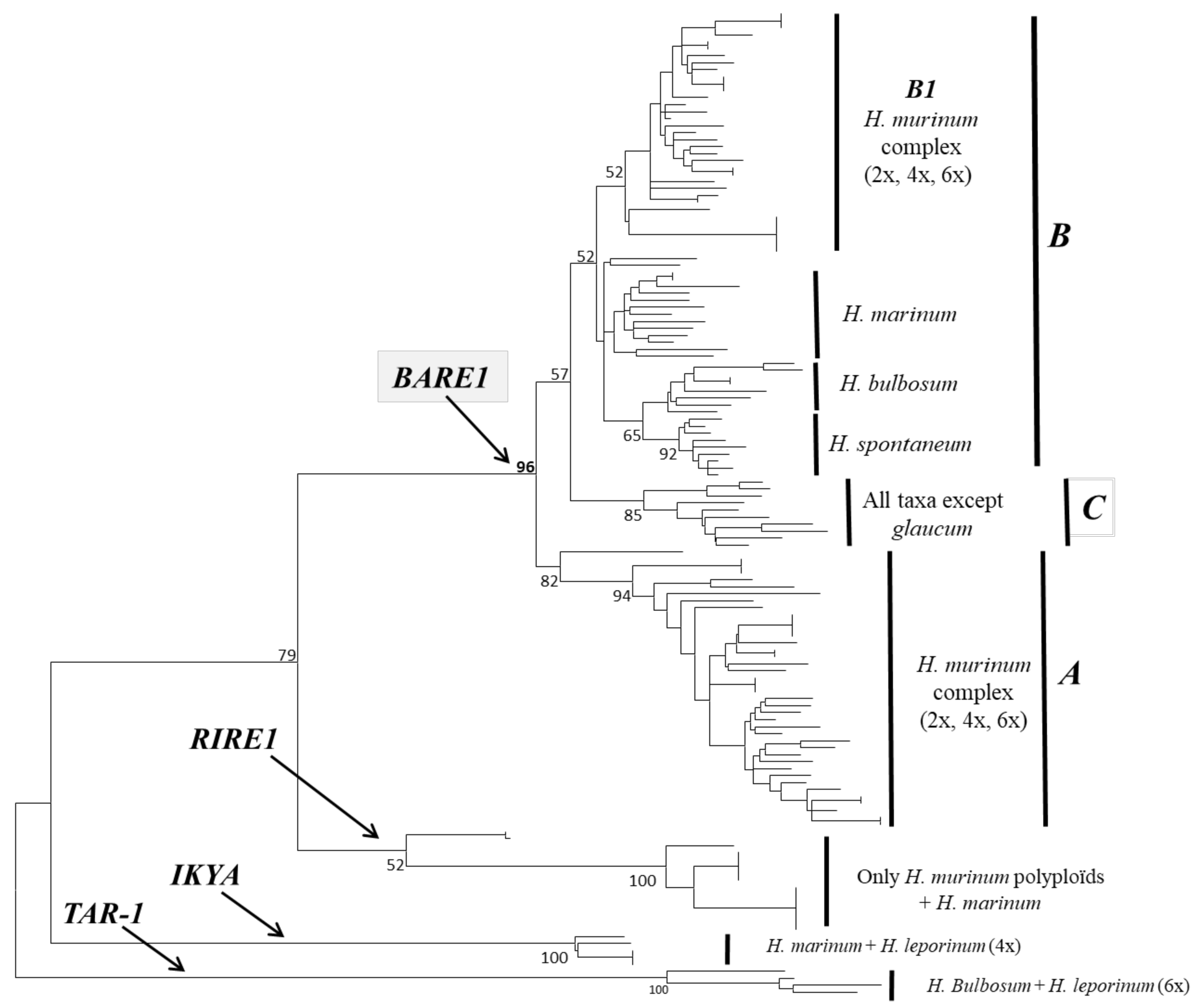


Fig.3

spontaneum / bulbosum (BARE1-B)

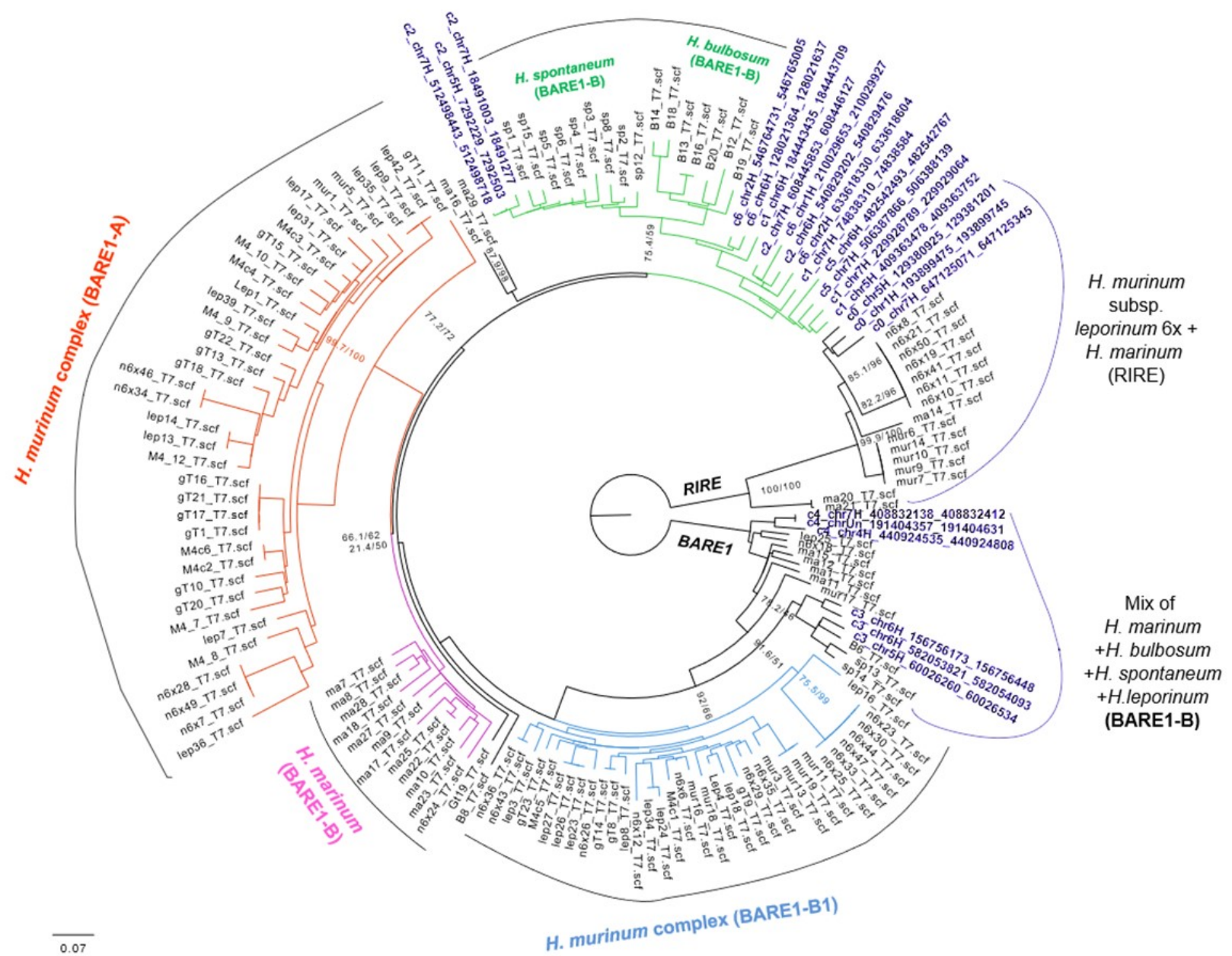


Fig.4

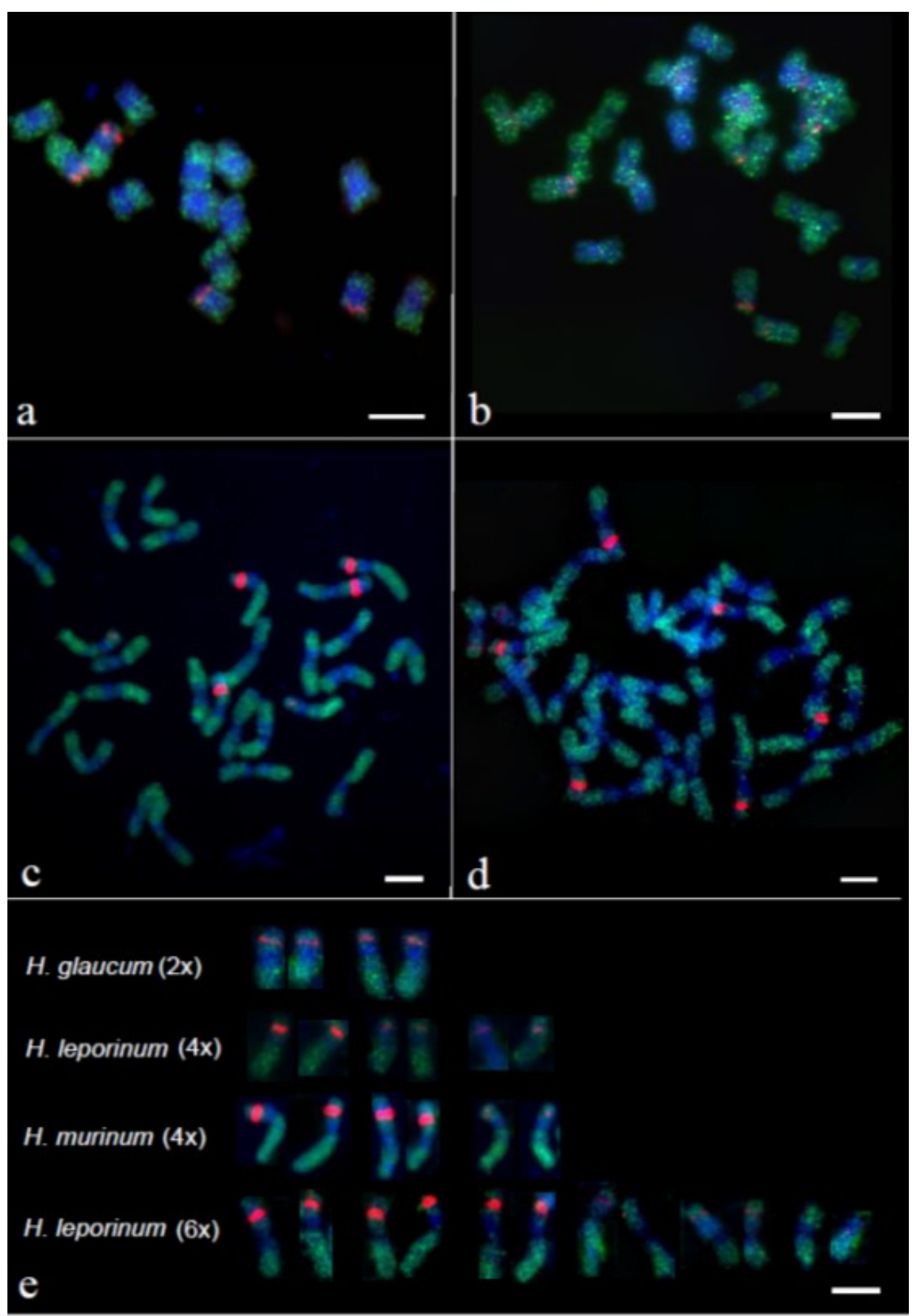


Fig.5

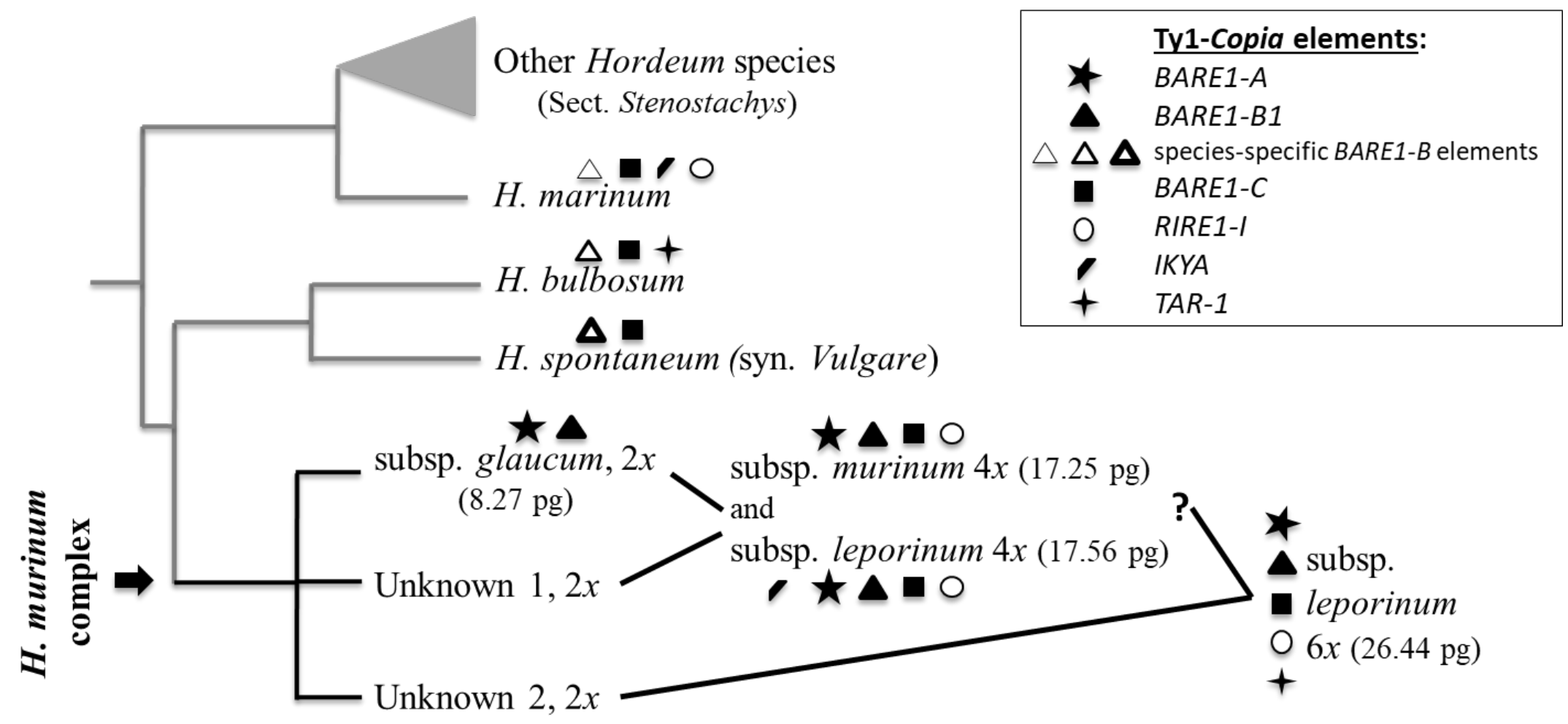

